\title{
Influence of diet, vitamins and chemotherapeutic agents on gastrointestinal cancer
}

\author{
JOSEPH C KOLARS` AND CANDACE L KURTH ${ }^{\dagger}$
}

*Shanghai Second Medical University, Shanghai, China and ${ }^{\dagger}$ University of Michigan, Ann Arbor, USA

\begin{abstract}
Dietary influences play a major role in the pathogenesis of most gastrointestinal malignancies. However, it has been difficult to define which dietary components will be most significant for any given individual. In this article we discuss the methodological challenges to research in this field as well as recent observations that have been made on the role of dietary factors in specific digestive tract neoplasms.
\end{abstract}

Key words: cancer, chemoprevention, cytochrome P450, diet, digestive tract, neoplasms, xenobiotics.

\section{INTRODUCTION}

Dramatic advances in our understanding of the molecular events associated with the pathogenesis of gastrointestinal cancer have taken place over the past 5 years. The impact of environmental factors on these events have been difficult to elucidate, in part due to the complex interactions that occur during the transformation from normal to malignant mucosa. It is estimated that one-third of all malignancies are related to our diet, with estimates as high as $85 \%$ for malignancies of the alimentary canal. ${ }^{1}$ Several important observations confirm the importance of the environment on gastrointestinal cancer, most of which are based on changes in cancer incidence when individuals move from one area of risk to another. Perhaps the most classic of these is based on the observation that residents of Japan, where the relative risk of stomach cancer is high and colonic cancer low shifts towards that of the local population when they move to the West where the relative risk of colon cancer is high and stomach cancer is low. Similar observations have also been made in migrants from China. ${ }^{2}$ Although the role of diet is unrefutable, the identification of specific dietary factors has been harder to define.

\section{METHODOLOGICAL CONSIDERATIONS}

The influence of diet on cancer is of enormous interest to the general public with frequent coverage of new findings in the lay press. The focus and pertinence of these studies are often not adequately discussed. The available data regarding the relationship between diet and carcinogenesis are at times conflicting due, in part, to the following limitations.

1. Mechanistic studies performed in laboratory animals or in ex vivo biological systems contribute valuable hypotheses for testing in humans but are often not predictive of what may take place in population-based studies.

2. Intraluminal factors (e.g. bile acid concentrations) as well as mucosal factors (e.g. P450 enzyme expression, molecular genotype) differ between individuals and compromise our ability to reliably use animal models to define the role of specific dietary components.

3. Population studies are time-consuming and expensive. A change in the rate of gastrointestinal cancer would likely not be realized for a prolonged interval (e.g. 10 years) after the onset of a sustained dietary

Correspondence: Joseph C Kolars, Shanghai Second Medical University, Shanghai Center Ste 203W, 1376 Nanjing Xi Lu, Shanghai 200040, China. Email: <kolars@uninet.com.cn>

Presented at the Third Asian AGA Course on Gastroenterology, Alimentary Disease Week, 12-17 December 1997, Hong Kong, China. 
intervention. Surrogate markers of malignancy (e.g. cellular hyperproliferation) have been of assistance in selecting specific dietary interventions that are worthy of more prolonged trials.

4. The complex interplay of dietary components makes it difficult to alter one component without affecting the relative presence of other dietary constituents. When a specific dietary component is altered, a compensatory change often takes place with other dietary constituents. Thus, identification of the component with the primary effect can be quite challenging.

5. Specific micronutrients contained within a food or diet are often difficult to identify and highly variable.

Conclusive scientific evidence that can be used to positively influence the health of the community is currently quite limited. We would like to have evidence based on high-quality case-control or cohort epidemiological studies from which we can test hypotheses that may help to define the biological events that are taking place. However, most studies to date provide speculation based on population-based observations or, more commonly, carcinogenesis in laboratory models. It is imperative that we understand the numerous challenges and potential flaws inherent to these approaches.

\section{DIETARY MACRONUTRIENTS}

Protein, fat and carbohydrates make up the major components of our diets and, as such, are often referred to as macronutrients. Several randomized controlled studies have demonstrated the positive correlation between dietary animal fat and carcinoma of the colon. ${ }^{3}$ More recently, it would appear that this risk is best associated with the ingestion of red meat. When reviewing studies on dietary macronutrients, it is important to keep in mind that to compare diets that are isocaloric, changes in one macronutrient group are invariably associated with compensatory changes in one of the other groups. For example, it has been advocated in the West that dietary fat should be reduced to levels of $30 \%$ of total calories with hopes of realizing a decrease in cardiovascular disease and some malignancies (colon, and possibly breast or prostate). If such a decrease was observed, could we in fact attribute this to a reduction in dietary fat or to the increase in carbohydrate that is required to maintain the same level of caloric intake? This problem was perhaps best illustrated by the early observation by Burkitt and colleagues of a reduced incidence of colon cancer in Africa where the diet is high in fibre., 4 More recent findings would suggest that while of probable benefit in reducing the risk for colon cancer, a diet high in dietary fibre is usually a marker of relatively low animal fat intake which likely has greater influence on reducing the risk of colorectal cancer than does high fibre intake. ${ }^{6}$

\section{DIETARY MICRONUTRIENTS}

The majority of studies regarding diet and cancer focus on micronutrients in the diet: pesticides, minerals, vit- amins, and other xenobiotics present in relatively small quantities that may have either causative or protective effects with regard to gastrointestinal malignancy. These components are often difficult to measure and are highly variable by region, season or crop. Synthetic pesticides have not been demonstrated to play a significant role in gastrointestinal malignancies. This is not surprising when one considers that most plants produce 'natural' pesticides to facilitate survival. These 'natural' pesticides are in far greater abundance than synthetic pesticides. It is estimated that the average daily American diet contains approximately $0.09 \mathrm{mg}$ of synthetic pesticides relative to $1500 \mathrm{mg}$ of 'natural' pesticides produced by the plants themselves. ${ }^{7}$

The role of vitamins in reducing the risk of gastrointestinal malignancy is at best controversial, particularly when taken as supplements to a well-balanced diet. While diets containing fruits and vegetables are almost always associated with lower risks of gastric and colorectal cancer, attempts to duplicate these benefits with supplements of vitamin A, C, E or beta-carotene have generally been unsuccessful. In fact, supplements alone may potentially increase the risk of malignancy. In a randomized, double-blind, placebo-controlled primary prevention trial involving 18314 smokers and workers exposed to asbestos, vitamin A and beta-carotene supplements were associated with an increased risk of death from all causes including lung cancer and cardiovascular disease. ${ }^{8}$ The chemical complexity of diets prevents the easy assignment of benefit or risk to any one nutrient. Furthermore, careful distinctions must be made between reducing dietary deficiencies that may be linked to a gastrointestinal cancer risk (e.g. selenium) and realizing a benefit from supplementing a diet that is not obviously deficient.

An often overlooked mechanistic consideration involves the role of dietary components on mechanisms by which carcinogens are metabolized. Dietary carcinogens are, in general, lipophilic and ingested as procarcinogens which must undergo oxidative metabolism, usually by cytochrome P450 (CYP) enzymes, creating a reactive metabolite which can form strategic DNA or protein adducts resulting in carcinogenesis. The CYP enzymes are substrate specific and variably expressed in organs with a wide variability among individuals. ${ }^{9}$ This variability undoubtedly influences the risk an individual has of bioactivating a carcinogen as well as the organ which is affected. The potential influence of CYP enzyme activity on dietary procarcinogens is perhaps best demonstrated when observing the pharmacokinetics of medications when taken with various diets. For example, it has been observed that the ingestion of a long list of medications with grapefruit juice will have a positive increase on oral bioavailability. Watkins and colleagues $^{10}$ demonstrated that grapefruit juice downregulates CYP3A4 enzymes within the mucosa of the small intestine resulting in decreased first-pass metabolism at the site of drug absorption and increased blood levels. Of note, this same enzyme is responsible for metabolizing a long list of drugs and xenobiotics including one of the best-recognized dietary carcinogens, aflatoxin B. It is interesting to speculate that dietary induced changes in CYP3A4 may, in fact, alter one's 
risk for aflatoxin B-related hepatocellular carcinoma. ${ }^{11}$ Undoubtedly, other dietary factors place a significant role in altering phase 1 oxidation enzymes, which subsequently influence the molecular chain of events resulting in carcinogenesis. It is worth noting that studies such as these require adherence to a carefully controlled diet with close documentation of the CYP expression pre- and post-dietary intervention.

\section{HEPATOCELLULAR CARCINOMA}

Studies by Ozturk and others have clearly demonstrated the role of aflatoxin B as a cofactor for hepatocellular carcinoma in patients with underlying hepatic inflammation such as that which occurs with chronic hepatitis. ${ }^{12}$ Metabolism of dietary aflatoxin B by CYP enzymes results in production of a reactive aflatoxin $B$ metabolite which can form specific adducts in strategic hot-spots, such as codon 249 in the $p 53$ tumour suppression gene. Of note, CYP3A, the most important enzyme responsible for this bioactivation, is the predominant CYP enzyme in human hepatocytes and enterocytes. $^{9}$

The field of hepatic malignancy chemoprevention is populated with anecdotal evidence of beneficial concoctions with a paucity of meaningful trials that support their efficacy. One intriguing possibility that has been explored in a randomized controlled study demonstrates that polyprenoic acid, a synthetic retinoid, significantly reduced the occurrence of second primary hepatomas. ${ }^{13}$ In the future, we will also have the results of an ongoing phase II trial in Qidong, China, on the potential beneficial effect of olitipraz, a synthetic dithiolthione similar to those found in cruciferous vegetables, on preventing hepatocellular malignancy (B Levin pers. comm., 1997).

\section{COLORECTAL MALIGNANCIES}

Colorectal cancer is the number two malignancy in the West and rapidly increasing in the East. In Shanghai, the incidence of colon cancer has risen by $75 \%$ between 1972 and $1989 .{ }^{14}$ Table 1 summarizes the relative role of known dietary interventions for colorectal carcinoma with those in the definite category supported by multiple, carefully performed, population-based studies. The interventions noted in the definite category have been demonstrated to alter the risk by two- to three-fold when comparing low and high use groups. However, it is not clear that these benefits are additive if each beneficial intervention was pursued together with the others.

Over 30 studies have looked at the relationships of fruits and vegetables in protecting against colorectal malignancies. However, we have yet to understand the specific agents or their role in influencing carcinogenesis.

A number of observational studies have confirmed a beneficial role for non-steroidal anti-inflammatory drugs such as aspirin in reducing the risk of mortality from colon cancer by $40-50 \%$ at doses as low as $325 \mathrm{mg}$ every other day. ${ }^{15}$ These studies have also been supported by a number of trials showing regression of adenomatous polyps on sulindac. The mechanism of this apparent chemopreventive effect is unknown but likely involves inhibition of cyclo-oxygenase 1 and 2 as well as altered cytokine expression..$^{15-17}$

Fibre has received a great deal of attention, particularly in the early observations of Burkitt who observed an association between high faecal bulk and low cancer rates in Africa. As noted earlier, some of the initial enthusiasm for fibre was probably due to the reduced levels of animal fat in diets containing high fibre. However, a meta-analysis of 60 studies suggests that a high-fibre diet is protective against colon cancer. ${ }^{18}$ Animal studies suggest that the less fermentable and more insoluble fibres (e.g. cellulose, lignins) are likely to be the most protective with wheat bran having the most consistently positive effect. Potential mechanisms of action include lowered $\mathrm{pH}$, increased short-chain fatty acids, decreased transit time, adsorption of bile acids, and dilution of colonic contents. ${ }^{5}$

Energy balance has been demonstrated to be an important risk factor for colorectal malignancy. This is likely to be of substantial importance in the West where it is estimated that $25 \%$ of individuals are overweight. The relative contribution of physical activity, body mass index (BMI), and caloric intake has been recently defined by Slattery et al. who compared a cohort with colorectal cancer with a carefully matched control group to study the relationship of these variables. ${ }^{19}$ Individuals with high physical activity, low caloric intake, and low BMI were defined with a relative risk of 1.0 and compared with individuals with low physical activity, high caloric intake, and high $\mathrm{BMI}$ who had a rela-

Table 1 Nutritional and chemopreventive interventions to reduce colorectal carcinoma

\begin{tabular}{llll}
\hline Definite & Probable & & Possible \\
\hline Fruits/vegetables & Fibre & Curcumin (tumeric) & Polyphenols (ellagic acid) \\
Energy balance & Garlic & Carotenoids & Dithiolthiones \\
Non-steroidal anti-inflammatory drugs & DFMO & Vitamin C & Selenium (when deficient) \\
Reduction of animal fat/red meat & Reduction of & Vitamin E & Oestrogen \\
& excess ETOH & Vitamin A & Calcium \\
\hline
\end{tabular}

DFMO, difluoromethylornithine; ETOH, ethanol. 
Table 2 Dietary factors related to stomach cancer

\begin{tabular}{ll}
\hline Decreased risk & \multicolumn{1}{c}{ Increased risk } \\
\hline Fruit & Salted/smoked foods \\
Vegetables, particularly & Pickled/preserved foods \\
allium vegetables: & Heterocyclic amines \\
$\quad$ Onions & Ethanol \\
Garlic & \\
Leeks & \\
Chives & \\
\hline
\end{tabular}

tive risk of 3.4. When stratifying each of these variables, physical activity was the most important determinant of risk and greatly modified the increased risk associated with high caloric intake or BMI. Thus, physical activity seems to be the most important component of 'energy balance' in colorectal carcinogenesis.

\section{UPPER GASTROINTESTINAL TRACT MALIGNANCIES}

A list of dietary factors associated with carcinoma of the stomach are shown in Table 2 . As with colorectal malignancies, ingestion of fruits and vegetables is consistently associated with a reduction in the risk of stomach cancer. Allium vegetables (e.g. onions, garlic, leeks) have been shown in several case-controlled studies to reduce the risk of stomach cancer, probably due to their organosulphur and flavonol compounds with odds ratios between 0.3 and $0.9 .^{20}$ In a large cohort study involving 120852 adults in The Netherlands, ingestion of onions ( $\geq 0.5$ onions/day) was associated with a reduced risk of carcinoma in the non-cardia region of the stomach ( $R R=0.50,95 \% \mathrm{CI}=0.26-0.95$ ) during 3.3 years of follow up. Consumption of leeks or garlic was not associated with a reduction in risk. ${ }^{20}$

Oesophageal carcinoma is associated with smoking and alcohol in the West but relative dietary deficiencies likely account for the higher incidence of adenocarcinoma of the proximal gastrointestinal tract that is observed in the East. A recent phase III study involving almost 30000 adults was completed in the LinXian Province of China, an area with a high incidence of adenocarcinoma of the lower oesophagus and proximal stomach. A significant reduction of malignancy in patients supplemented with beta-carotene, vitamin $\mathrm{E}$ and selenium ( $R R=0.91,95 \% \mathrm{CI}=0.84-0.99)$ was observed, particularly stomach cancer. ${ }^{21}$ Two other trials in China involving patients with dysplasia of the proximal gastrointestinal tract failed to demonstrate a significant reduction in subsequent cancer or mortality when supplemented with a variety of vitamins and minerals. ${ }^{22,23} \mathrm{~A}$ preliminary study in China also suggests that tea may offer some protection against precancerous gastric lesions. ${ }^{24}$

\section{CONCLUSION}

A growing number of high-quality population-based studies are providing us with a better understanding of the dietary interventions which can reduce our risk of gastrointestinal malignancies. The development of reliable surrogate biomarkers for these cancers and methods to correlate gentotypic factors present in a study population will greatly enhance our ability to determine which dietary components to pursue or avoid. Based on the latter, it is quite likely that these will vary depending on the individual. Meanwhile, we must be careful to avoid overstating what we know or believe to the population that demands of us a checklist of good and bad dietary ingredients.

\section{REFERENCES}

1 Doll R, Peto R. The causes of cancer: Quantifiable estimates of avoidable risks of cancer in the United States today. I. Natl Cancer Inst. 1981; 66: 1191-308.

2 Levin B. Nutrition and colorectal cancer. Cancer 1992; 70: 1723-6.

3 Giovannucci E, Willett WC. Dietary factors and risk of colon cancer. Ann. Med. 1994; 26: 443-52.

4 Klurfeld DM. Dietary fiber-mediated mechanisms in carcinogenesis. Cancer Res. 1992; 52: S2055-9.

5 Sandler RS. Epidemiology and risk factors for colorectal cancer. Gastroenterol. Clinics North Am. 1996; 25: 717-35.

6 Boland CR, Kolars JC. Fiber and colon cancer: The weight of the evidence. Gastroenterology 1992; 103: 1964-7.

7 Gold LS, Slone TH, Stern BR, Manley NB, Ames BN. Rodent carcinogens: Setting priorities. Science 1992; 258: 261-5.

8 Omenn GS, Goodman GE, Thornquist MD et al. Effects of a combination of beta carotene and vitamin $A$ on lung cancer and cardiovascular disease. N. Engl. J. Med. 1996; 334: 1150-5.

9 Kolars JC, Schmiedlin-Ren P, Schuetz JD, Fang C, Watkins PB. Identification of rifampin-inducible P450IIIA4 (CYP3A4) in human small bowel enterocytes. f. Clin. Invest. 1992; 90: 1871-8.

10 Lown KS, Bailey DG, Fontana RJ et al. Grapefruit juice increases felodipine oral availability in humans by decreasing intestinal CYP3A protein expression. 7. Clin. Invest. 1997; 99: 2545-53.

11 Kolars JC, Benedit PE, Schmiedlin-Ren P, Watkins PB. Aflatoxin B1-adduct formation in rat small bowel enterocytes. Gastroenterology 1994; 106: 433-9.

12 Ozturk M. P53 mutation in hepatocellular carcinoma after aflatoxin exposure. Lancet 1991; 338: 1356-9.

13 Muto Y, Moriwaki H, Adachi S et al. Prevention of second primary tumors by an acyclic retinoid, polyprenoic acid, in patients with hepatocellular carcinoma. N. Engl. Y. Med. 1996; 334: 1561-7.

14 Devesa SS, Jun F, Zheng W, Blot WJ, Fraumeni JF et al. Rising incidence of colon cancer in Shanghai. Cancer Epidemiol. Biomarkers Prev. 1993; 2: 293-4.

15 Krishnan K, Brenner DE. Chemoprevention of colorectal cancer. Gastroenterol. Clinics North Am. 1996; 25: 821-58. 
16 Potter JD, Slattery ML, Bostich RM, Gapstur SM. Colon cancer: A review of the epidemiology. Epidemiol. Rev. 1993; 15: 499-545.

17 Estabrook R, the Committee on Comparative Toxicity of Naturally Occurring Carcinogens. Carcinogens and Anticarcinogens in the Human Diet. Washington, DC: National Academy Press, 1996.

18 Trock B, Lanza E, Greenwald P. Dietary fiber, vegetables, and colon cancer: Critical review and meta-analyses of the epidemiologic evidence. I. Natl Cancer Inst. 1990; 82: 650-61.

19 Slattery ML, Potter J, Caan B et al. Energy balance and colon cancer: Beyond physical activity. Cancer Res. 1997; 57: $75-80$.

20 Dorant E, Van Den Brandt PA, Goldbohm RA, Sturmans F. Consumption of onions and a reduced risk of stomach carcinoma. Gastroenterology 1996; 110: 12-20.

21 Blot WJ, Li J, Taylor PR, Guo W et al. Nutrition interven- tion trials in Linxian, China: supplementation with specific vitamin/mineral combinations, cancer incidence, and disease specific mortality in the general population. $\mathcal{~}$. Natl Cancer Inst. 1993; 85: 1483-92.

$22 \mathrm{Li} \mathrm{J}$, Taylor PR, Li B et al. Nutrition intervention trials in Linxian, China: multiple vitamin/mineral supplementation, cancer incidence, and disease-specific mortality among adults with esophageal dysplasia. f. Natl Cancer Inst. 1993; 85: 1492-8.

23 Munoz N, Bang LJ, Day NE et al. Effect of retinol, zinc, riboflavin on the prevalence of precancerous lesions of the esophagus: randomized, double-blind intervention study of high risk populations in China. F. Natl Cancer Inst. 1987; 79: 687-91.

24 Chen JS, Wong BCY, Wu YN et al. Preliminary analysis of dietary risk factors for precancerous gastric lesions in population of Changle, a high risk area of gastric cancer in China (abstract). Gastroenterology 1997; 112: A547. 